\title{
La fragmentación del conocer en educación física \\ The fragmentation of knowledge in physical education
}

*Sebastian Peña Troncoso, **Sergio Toro Arévalo, ***Jaime Cárcamo Oyarzún, ****Claudio Hernández Mosqueira, $* * * * *$ Mauricio Cresp Barría

*Universidad Austral de Chile (Chile)/Universidad SEK (Chile), **Universidad de Santiago de Chile (Chile)/Universidad Austral de Chile (Chile), $* * *$ Universidad de la Frontera (Chile), $* * * *$ Universidad de los Lagos (Chile)/Universidad Adventista de Chile (Chile), *****Universidad Católica de Temuco (Chile)

Resumen. El estudio tuvo como objetivo evaluar con fines diagnósticos la formación conceptual en la asignatura de Educación Física, en estudiantes chilenos de enseñanza media. El estudio fue metodológicamente de corte descriptivo transversal. Participaron 659 estudiantes, $54 \%$ (hombres) y $46 \%$ (mujeres), con edades entre los 13 y 14 años, de las tres dependencias educativas del país; municipal (36.4\%), subvencionado (37.8\%) y privado (25.8\%). Se realizó un muestreo probabilístico estratificado según dependencia educativa y sexo de los estudiantes. Para la recolección de los datos se aplicó un cuestionario diseñado y validado con estudiantes del currículo chileno. Los principales resultados indican que existen diferencias estadísticamente significativas entre las dependencias educativas, con resultados específicos de la prueba Anova de $f=48.584 p<.05$ a favor de los colegios particulares. En conclusión, el estudio muestra un bajo dominio disciplinar de los alumnos y alumnas en las diferentes dependencias educativas, manifestando, una vez más, la necesidad de buscar metodológicas innovadoras para la disciplina, en función de mejorar los resultados de la dimensión conceptual.

Palabras Claves: Formación conceptual; contenidos curriculares; educación física, evaluación.

Abstract. The study aimed at diagnosing the conceptual formation in physical education in Chilean high school students. The study used a descriptive cross-sectional methodology. A total of 659 students participated, 54\% boys and 46\% girls, aged between 13 and 14 years old, from the three educational units in the country; public schools (36.4\%), subsidized schools (37.8\%) and private schools (25.8\%). A stratified probabilistic sampling was performed based on students' educational dependence and gender. For the data collection, a questionnaire designed and validated with students of the Chilean curriculum was applied. The main results indicate that there are statistically significant differences between educational units, with specific results from Anova of $f=48,584 \mathrm{p}<.05$ in favor of private schools. In conclusion, the study shows a low disciplinary domain of the students in the different educational units, highlighting once more the need to seek innovative methodologies within this discipline in order to improve the learning of its conceptual dimension. Keywords: Conceptual formation; curricular contents; physical education; evaluation.

\section{Introducción}

La enseñanza de la Educación Física (EF) en el contexto escolar, por años ha sido una de las asignaturas que ha fragmentado el conocimiento, dando un marcado privilegió y énfasis a las habilidades o procedimientos, aun sabiendo que, el conocimiento, desde una perspectiva sistémica y compleja (Sergio, 2019; Moreno, Toro y Gómez, 2020) no es posible fraccionarlo, pero si profundizar o ampliar. Sin embargo, al revisar las diferentes evaluaciones de los escolares en EF, existe una tendencia hacia la evaluación procedimental (saber hacer) a través de la realización de test físicos, controles y pruebas de ejecución, dejando de lado o al menos omitiendo, la formación actitudinal y conceptual (Peña, Toro, Osses, Moreno \& Hernández-Mosqueira, 2019). Esta última dimensión «formación conceptual» se identifica con el «saber», siendo la información que podemos recordar exactamente como fue memorizada (Mcpherson, 1994; Thomas \& Thomas, 1994). La característica principal es que el alumno lo puede verbalizar y declarar (Ruiz, Sánchez, Durán \& Jiménez, 2006) o que se conforma por medio del lenguaje (Díaz-Barriga \& Hernández, 2010). Lo valioso de considerar la dimensión conceptual, es que le permite a los estudiantes que sean capaces de comprender y declarar lo aprendido, y no funcionen como meros agentes que repiten una acción que determina el profesor o profesora.

Esta fragmentación ha llevado a una comprensión erró-

Fecha recepción: 14-01-20. Fecha de aceptación: 16-06-20 Sebastián Peña Troncoso sebap988@hotmail.com nea, sino parcial, que tiene la población frente a la educación física (Moreno, Campos \& Almonacid, 2012). Lo que es peor aún, los directivos de los establecimientos educacionales, se están interesando por una educación física orientada al rendimiento físico y deportivo, al fomento y desarrollo de talentos deportivos al extremo de solicitar a los docentes que mejoren los resultados performáticos del estudiantado, la conformación de selecciones deportivas en deportes tradicionales y, por tanto, a procesos de selectividad y exclusión. Tal situación continúa con la visión de una asignatura biomédica, mecánica (Rodríguez, \& Hernández, 2018; Tobar, Gaete, Lara, Pérez, \& Freundt, 2019) basada en la tecnificación del aprendizaje (Cardozo, Velasco, Domínguez, \& Reyes, 2019) orientada a evaluar el rendimiento de la condición física (Moreno \& Medina, 2012; Zaragozá \& Martínez, 2018) que, por lo demás, dada su mínima cantidad y regularidad, tienen un bajo impacto en la salud y los procesos de enseñanza y aprendizaje de los estudiantes (Peña, Osses, Navarro \& Beltrán, 2017). Aun así, a los colegios les interesa continuar con estas dinámicas, ya que les permite obtener buenos resultados en las mediciones nacionales (Rodríguez, Coz, Durán, Guajardo, Alvarado \& Doña, 2015). Autores como Rodríguez-Rodríguez et al., (2016) y García (2016) señalan que la disciplina necesita una evaluación integral, pero, además que el docente disponga de un dominio de la disciplina apto para la enseñanza, mediante la utilización de estrategias, recursos didácticos y evaluaciones adecuadas, para un contexto determinado (Beltrán-Véliz, Navarro \& Peña, 2018).

De igual forma, diferentes estudios como los de Carmona (2007); Díaz (2012); Domínguez, Arroyo, Gallego, González \& 
del Villar (2006); Hernández, Velázquez \& Martínez (2007); Ibáñez (2005); Otero, González, Calvo \& Molina (2012) señalan que la evaluación en EF se ha centrado tradicionalmente en las habilidades, dicho de otra manera preocupados del saber hacer, aun cuando el conocimiento y las actitudes es crucial para el perfeccionamiento del proceso pedagógico de niños y jóvenes (Aleixo \& Mesquita, 2016). Desde este plano, la evaluación en EF, se encontraría arraigada a la escasa significación pedagógica de la disciplina y a su constante predominancia orgánica, dualista, técnica y objetivada del movimiento (Almeida, Bracht, \& Vaz, 2012; Bracht, 2012; Escudero, 2011; Gallo, 2008; Whitehead, 2010). Perpetuando de esta manera, una EF que mantiene la idea de las clases como modelación, normalización, instrucción y disciplinamiento (Gallo \& Martínez, 2015). En este sentido, es necesaria una reformulación de la disciplina, que pueda obedecer a la pluralidad de formas de educar en EF (Páez y Hurtado, 2019) en ambientes de enseñanza donde se favorezca la confianza, responsabilidad y la autonomía (Vera \& Moreno, 2016). Pero sobre todo, es necesario develar el carácter epistémico de la disciplina.

Existen diversos factores que pueden explicar, porque los docentes han ignorado en la práctica el desarrollo de contenidos de carácter conceptual y actitudinal y, por tanto, la exigencia de los correspondientes aprendizajes. Uno de ellos, lo constituye la baja cantidad de horas semanales que han tenido los docentes en su práctica laboral (Peña \& Hernández, 2014). El otro, posiblemente de mayor peso, radica en la concepción hegemónica de esta disciplina que le atribuye al desarrollo del rendimiento motor del alumnado su razón de ser y su objeto principal, lo que supone tratar de obtener el mayor tiempo posible de compromiso motriz durante el desarrollo de las clases (Hernández, Velásquez \& Martínez, 2007). En efecto, la acción conjunta de ambos factores ha determinado que, a lo largo de la historia de la EF, la preocupación de los profesores e investigadores haya estado centrada siempre en la práctica motriz, orientados al rendimiento deportivo (González, García, Pastor \& Contreras, 2011; Méndez, 2005) y más concretamente, en la variable tiempo de movimiento (Gusthart, Kelly \& Rink, 1997; Metzler, 1989; Silverman, Devillier \& Ramírez, 1991). Asimismo, el concepto de eficacia docente ha estado asociado al rendimiento que obtienen los estudiantes, valorado a través de pruebas estandarizas, tal como el Sistema de Medición de la Calidad de la Educación (SIMCE). Esta prueba está basada en la conocida batería de test Eurofit y, ha sido estos últimos años la herramienta de evaluación nacional en la asignatura de EF del currículo de Chile. Fue aplicado por primera vez como piloto el año 2010 a 13.585 estudiantes de Educación General Básica (EGB) correspondientes al último año de este ciclo, $8^{\circ}$ Básico (13 -14 años), distribuidos en 335 centros educativos a nivel nacional (Agencia de la Calidad de la Educación, 2013). Sin embargo, estas pruebas hace cinco años que no se aplican.

Esta medida puede resultar interesante si se evaluara de manera diagnóstica o formativa, otorgándole un carácter pedagógico. No obstante, los datos recogidos se analizan, se comparan y se establecen ciertos puntos de referencia que permiten clasificar a los colegios en diferentes categorías según sus resultados, logrando de esta manera estigma- tizar los establecimientos entre buenos y malos, generando una competitividad orientada al rendimiento y no al aprendizaje. Sin duda, estas prácticas pueden llegar a transformarse en uno de los mayores problemas de cualquier medición pública, cuyos objetivos suelen ser clasificatorios, generando segregación, discriminación y desmotivación en la población escolar (Rodríguez et al., 2015). Sería una propuesta interesante si, a partir de los datos obtenidos se detectasen grupos poblacionales problemáticos y se establecieran propuestas de intervención para abordarlos. Desde esta mirada, el SIMCE ha sido fuente de críticas por diferentes profesionales de la salud y la educación. Una de los principales cuestionamientos es el escaso impacto en la salud de los educandos. Al respecto, Devís \& Peiró (1993) plantean dos importantes críticas en relación a los aportes que nos pueden entregar estos test en la salud de nuestros estudiantes. Por un lado, nula utilización de indicadores asociados directamente con la salud, tales como: la presión sanguínea o lípidos en la sangre; y, por otro lado, no existe correlación entre los resultados obtenidos por los jóvenes en los test de condición física y su salud en la vida adulta. Aun así, muchos de los profesores de EF actualmente siguen priorizando una variedad de test físicos en sus clases.

Autores, como Zabalza, Viciana \& Lozano (2002) señalan que, los profesores de EF priorizan el aprendizaje de la disciplina otorgando una clara importancia a las habilidades sobre los conceptos y actitudes. Al respecto, López \& Moreno (2002) en un análisis de la enseñanza de estos contenidos en educación física señalan que uno de los problemas de los docentes del área es el desconocimiento de cómo llevar los conocimientos teóricos a las clases de EF. Señalan una importante deficiencia en la «formación inicial» como causa y, definieron tres perfiles docentes en relación con este aspecto. En primer lugar, aquellos que afrontan su incapacidad con un inmovilismo basado en continuar con la metodología tradicional. Otra corriente basa el aprendizaje de hechos y conceptos en las clases teóricas y evaluaciones escritas. La tercera tendencia fundamenta el proceso de enseñanza y aprendizaje conceptual en los libros de texto. En este sentido, y siguiendo lo planteado por López et al., (2016) es importante que la formación del profesorado de EF tenga un enfoque más competencial y práctico de lo que ha tenido tradicionalmente, entendiendo que una competencia implica la aplicación integrada de las capacidades básicas del saber, saber hacer, saber ser y saber estar. Es por ello, que es necesario revisar la formación de los futuros docentes de EF (Poblete, Linzmayer, Matus, Garrido \& Flores, 2018).

Desde el presente estudio, se reconoce la importancia del aprendizaje en el desarrollo de habilidades a través de la acción, pero, la crítica apunta a la necesidad de valorar la dimensión conceptual y actitudinal de la disciplina. Tanto para responder a los problemas motrices de la cotidianeidad, como también para desenvolverse en su entorno y comunidad, porque la experiencia del aprendizaje en EF debe nacer desde la incorporación de conocimiento hacia la ejecución, y a partir de una actitud, determinada por las situaciones o problemas que se enfrentan (Zabala, 2000). De esta manera, el instante más relevante del desarrollo intelectual se produce cuando lo teórico (contenidos conceptuales) converge con lo práctico y la experiencia (Castañer \& Trigo, 1995; 
Toro, Pazos, Vargas \& Vega, 2016), sin invisibilizar, los valores y las actitudes en cada acción desde el mismo proceso de aprendizaje. Desde esta perspectiva, la labor del profesor de EF es fundamental en el ámbito escolar (Moreno, Toro \& Gómez, 2020), ya que permite promover la autonomía y orientar la formación de sus escolares, aun así, Sánchez \& López (2019) mencionan que, la asignatura de EF es prioritariamente práctica, ya que esta se encuentra en consonancia con la singularidad de la disciplina.

En síntesis, pareciera ser que falta rigor profesional o reflexión sobre lo que significa educar en EF desde una multidimensionalidad, incorporando no solo las habilidades, sino que también, conceptos y actitudes, considerando los procesos culturales y sociales que pueden hacer de la clase no sólo una experiencia personal de vida activa, sino también una sociedad más cercana al buen vivir y cuidado por el entorno. Por esta razón, es necesario reconstruir la Educación Física Escolar con urgencia, empezando por la Formación Inicial del Profesorado (Trigueros, Rivera \& Moreno, 2020). Por lo tanto, consideramos relevante abordar un ámbito que tiene escasas investigaciones en Chile, por lo que el presente estudio tiene como propósito establecer un diagnóstico de la formación conceptual en EF de los estudiantes de segundo año de enseñanza media de la provincia de Llanquihue, Región de los Lagos, Chile.

\section{Método}

El enfoque del estudio fue de corte descriptivo, transversal (Ato, López \& Benavente, 2013).

\section{Muestra}

El presente estudio se realizó con estudiantes del segundo nivel de enseñanza media, considerando que en Chile la etapa secundaria es de cuatro años y la básica seis. Participaron 659 escolares, el 54\% hombres y $46 \%$ mujeres de las tres dependencias educativas del país, municipal (36.4\%), subvencionado (37.8\%) y privado $(25.8 \%)$, con edades que oscilaron entre los 15 y 18 años de edad. Para lograr la representatividad de la muestra se desarrolló un muestreo probabilístico estratificado según la dependencia administrativa y el sexo de los estudiantes (8 estratos). Según Scheaffer, Mendenhall \& Ott (1987), esta forma de muestrear, facilita la representatividad y permite obtener buenas estimaciones de los parámetros, permitiendo aumentar la cantidad de información, optimizando en gran parte los recursos disponibles.

\section{Instrumento}

Para la recolección de los datos se utilizó el Cuestionario de Aprendizajes declarativos Conceptuales en Educación Física (CADCEF), elaborado y validado, de acuerdo a los planes de estudio del primer y segundo nivel de enseñanza media ( 15 y 16 años de edad) del currículo chileno (PeñaTroncoso, Toro, Bustingorry \& Hernández-Mosqueira, 2018). En el aspecto formal, cabe señalar que las preguntas del instrumento se plantearon en forma de afirmaciones, siguiendo la estructura utilizada por Hernández, Velásquez \& Martínez (2007), de manera que cada estudiante logre precisar en cada afirmación si es correcta o incorrecta. Con el objeto de reducir el efecto de las posibilidades de acierto por elección al azar de cada respuesta, se apeló a la sinceridad de los estudiantes introduciendo cuatro opciones de respuesta, quedando estructuradas en: «No lo sé»; «Correcto»; «Tengo dudas»; «Incorrecto». Además, el instrumento constó de un segundo apartado, en donde se plantearon imágenes en función de la salud postural; en este caso, también se ofrecieron las mismas cuatro opciones de respuestas.

\section{Procedimientos}

La aplicación del instrumento fue realizada por los investigadores y, en algunos casos, por los profesores de EF de cada colegio, a los cuales se les capacitó para la aplicación del cuestionario. Se hizo hincapié en el anonimato de las repuestas y, en que contestaran con total sinceridad. Los cuestionarios fueron autoadministrados con presencia de los investigadores o profesores, es decir, se entregó a los participantes el cuestionario respectivo, el que debieron responder individualmente. El investigador o profesor podía apoyar al encuestado con cualquier problema relacionado con la interpretación del cuestionario. Las encuestas fueron aplicadas en la clase de EF. Para la realización de este estudio se solicitó la autorización de los directores de cada establecimiento, posteriormente, al ser oficializada dicha autorización y definidos los colegios en donde se aplicarían las encuestas, se informó a los padres de la metodología y el propósito del estudio mediante carta formal remitida por los investigadores, junto con un consentimiento informado para los padres.

Para participar en el estudio, los estudiantes tenían que entregar el consentimiento firmado por sus padres, madres o tutores y asistir al centro el día de la prueba, además, firmar un asentimiento informado. Es importante señalar que, para cumplir con los criterios éticos propuestos, se ha resguardado el anonimato de todos los y las estudiantes, y sólo se consideró el sexo, edad y dependencia administrativa, como datos demográficos de cada estudiante. Con ello, se respetaron los criterios éticos, de acuerdo a la declaración de Helsinki para el estudio con seres humanos (Manzini, 2000).

\section{Análisis estadísticos}

La distribución de los datos de las variables cuantitativas fueron valoradas a través de la prueba de KolmogorovSmirnov. Se realizaron cálculos estadísticos descriptivos de tendencia central (Media) y dispersión (Desviación Estándar) (DE). Para determinar si existen diferencias según sexo y dependencia administrativa, se aplicó el método T Student y Anova, con una prueba Post Hoc de Tuckey para identificar las diferencias significativas entre las variables evaluadas. En el caso de las variables cualitativas se realizaron asociaciones a través del coeficiente Chi-cuadrado de Pearson. Para todas las pruebas se determinó un nivel $p<.05$ para la interpretación de la significancia. El análisis de los datos descriptivos y comparativos se realizó con el programa estadístico IBM SPSS Statistics, versión 23.

\section{Resultados}

En la Tabla 1 se presentan los resultados relativos a la puntuación media y desviación típica obtenida a través de la 
sumatoria de respuestas correctas por el total de la muestra, en función de la dependencia administrativa y sexo de los estudiantes. La puntuación máxima se sitúa en 32 puntos y, por tanto, el valor medio (aprobado) en 16.

Considerando los resultados de cada dependencia administrativa, en función de la variable sexo, solo en los colegios particulares se producen diferencias significativas a favor de las mujeres, con resultados específicos de la prueba $T$ de $f=0.264 p<.05$. No obstante, en las dependencias municipales y subvencionados, no se producen diferencias estadísticamente significativas en función del sexo, con resultados específicos de la prueba $\mathrm{T}$ para los colegios municipales de $f=2.828 p>.05$; colegios subvencionados $\operatorname{de} f=$ $3.365 p>.05$.

Desde los resultados totales de cada dependencia educativa, y las diferencias producidas en función de la variable de respuestas correctas, se encontraron diferencias estadísticamente significativas, con resultados específicos de la prueba Anova de $f=48.584 p<.05$. Las diferencias se produjeron entre las dependencias municipales y subvencionados, municipales y particulares y, subvencionados y particulares, todos con un $(p=0.00)$

Con el objetivo de conocer el nivel de formación conceptual de cada dependencia administrativa, se decide recodificar la variable que determina la suma de respuestas correctas de cada estudiante, en función del percentil 75, donde el puntaje de corte se estableció en 17 puntos (Tabla 2). Estos resultados nos permiten identificar los estudiantes que están por sobre o bajo los 17 puntos. Se destaca que sólo 175 estudiantes del total de la muestra (659), se encuentran sobre el percentil 75.

La Tabla 3 muestra los porcentajes de respuestas correctas que están sobre y bajo el percentil 75, en función de cada dependencia administrativa. Se observa que existe una diferencia altamente significativa $p<.01$ a favor de los colegios particulares. En donde los estudiantes de colegios municipales solo superan los 17 puntos (12.5\%), los subvencionados en un (22.9\%) y los particulares el (51.8\%).

La segunda asociación (tabla 4) se establece entre las variables sexo y respuestas correctas por sobre y bajo el percentil 75 . Se observa que no existen estadísticamente diferencias significativas $p>.05$. En donde el $28.7 \%$ de los hombres supera los 17 puntos, mientras que las mujeres, sólo lo hacen en un $24.1 \%$.

\section{Discusión}

Existen diferentes evidencias empíricas como las de Hernández \& López (2007); Ibáñez (2005) y Velázquez, Hernández, Martínez \& Martínez de Haro (2009) que ratifican la importancia de la dimensión conceptual en EF, en una disciplina que por muchos años su principal orientación ha estado en la acción motriz a partir del «saber hacer». Precisamente, por esta concepción hegemónica de la disciplina orientada hacia las habilidades, es que la mayoría de las investigaciones en EF se enmarcan en el rendimiento deportivo (González, García, Pastor \& Contreras, 2011; Méndez, 2005), provocando que el desempeño físico y deportivo en los estudiantes sea sinónimo de «buena calificación». Esta situación histórica y tradicional ha permitido que los profesores
Tabla 1.

Respuestas correctas de cada dependencia educativa en función del sexo (Media y Desviación Estándar).

\begin{tabular}{lccc}
\hline & $\begin{array}{c}\text { Total } \\
\text { Media (DE) }\end{array}$ & $\begin{array}{c}\text { Hombres } \\
\text { Media (DE) }\end{array}$ & $\begin{array}{c}\text { Mujeres } \\
\text { Media (DE) }\end{array}$ \\
\hline Total & $13.49(4.96)$ & $13.85(4.82)$ & $13.08(5.10)$ \\
Municipal & $11.71(4.42)$ & $12.15(3.99)$ & $11.23(4.82)$ \\
Subvencionado & $13.30(4.72)$ & $13.85(5.13)$ & $12.83(4.31)$ \\
Particular & $16.28(4.81)$ & $15.70(4.69)$ & $17.51(4.86)$ \\
\hline
\end{tabular}

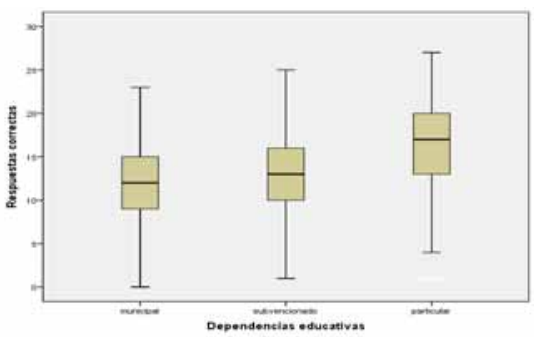

Figura 1. Diferencias de las respuestas correctas del cuestionario en función de cada dependencia educativa.

Tabla 2.

Percentiles de la variable respuestas correctas

\begin{tabular}{lcccc}
\multicolumn{5}{c}{ Percentiles de la variable respuestas correctas } \\
\hline \multicolumn{5}{c}{ Percentiles respuestas correctas } \\
\hline Respuestas correctas & $\mathbf{2 5}$ & $\mathbf{5 0}$ & $\mathbf{7 5}$ & $\mathbf{9 0}$ \\
Media & 10 & 13 & 17 & 20 \\
Desviación Típica. & 13.49 & & & \\
Minimo & 4.965 & & & \\
Máximo & 0 & & & \\
\hline & 27 & &
\end{tabular}

Tabla 3.

Asociación variable dependencia educativa y frecuencia de repuestas correctas sobre y bajo el percentil 75.

\begin{tabular}{lccc} 
el percentil 75. & \multicolumn{4}{c}{ Porcentajes Respuestas Correctas } \\
\hline Dependencia & Inferior & Superior & Total \\
\hline Municipal & 210 & 30 & 240 \\
& $87.5 \%$ & $12.5 \%$ & $100.0 \%$ \\
Subvencionado & 192 & 57 & 249 \\
& $77.1 \%$ & $22.9 \%$ & $100.0 \%$ \\
Particular & 82 & 88 & 170 \\
& $48.2 \%$ & $51.8 \%$ & $100.0 \%$ \\
Total & 484 & 175 & 659 \\
Chi-cuadrado de & $100.0 \%$ & $100.0 \%$ & $100.0 \%$ \\
Pearson= & 81.417 & \multicolumn{3}{c}{$\mathbf{p = 0 . 0 0 0}$} \\
\hline
\end{tabular}

Tabla 4.

Asociación variable sexo y frecuencia de repuestas correctas sobre y bajo el percentil 75.

\begin{tabular}{lccc}
\multicolumn{4}{c}{ Porcentajes respuestas Correctas } \\
\hline Sexo & Inferior & Superior & Total \\
\hline Hombres & 254 & 102 & 356 \\
& $71.3 \%$ & $28.7 \%$ & $100.0 \%$ \\
Mujeres & 230 & 73 & 303 \\
& $75.9 \%$ & $24.1 \%$ & $100.0 \%$ \\
Total & 484 & 175 & 659 \\
Chi-cuadrado de & $100.0 \%$ & $100.0 \%$ & $100.0 \%$ \\
Pearson= & 1.745 & \multicolumn{3}{c}{$\mathbf{p = 0 . 1 8 7}$} \\
\hline \multicolumn{4}{c}{}
\end{tabular}

Pearson=

de EF orienten sus clases bajo el principio de buscar el mayor tiempo posible de ejercicio físico, utilizando la evaluación sumativa como medio verificador de cuentas y medición de los resultados finales (Romero-Martín, Castejón-Oliva \& López-Pastor, 2015).

En relación a los resultados generales obtenidos, se visualiza una realidad caracterizada por el bajo conocimiento conceptual en EF y, en consecuencia, estos datos nos incitan a reflexionar sobre la necesidad de mejorar la calidad de los procesos de enseñanza y aprendizaje, con un especial enfoque en el sentido del porqué, de la ejecución de las actividades motrices, logrando que los estudiantes sean capaces de comprender, fundamentar y explicar sus propias acciones; de entender y analizar las repercusiones de dichas acciones sobre su propio cuerpo (Hernández \& López, 2007).

Los resultados a partir de las diferencias que existen en cada dependencia educativa, en función de la media de respuestas correctas obtenidas en el estudio, permite determinar, la existencia de diferencias significativas a favor de los colegios particulares, al igual que el estudio realizado por Cárcamo (2012). Estos resultados además, coinciden con los reportes de las políticas educativas y diferentes estudios 
(Bonilla et al., 2003; Mizala \& Romagera, 1998) que señalan que, el rendimiento académico considera múltiples variables como ingreso, educación de los padres y dependencia, determinando que los estudiantes que pertenecen a establecimientos particulares pagados tienen un mejor rendimiento académico. En este sentido, si bien existen varios factores que impactan a favor de los colegios privados, los resultados en general de todas las dependencias educativas demuestran un bajo bagaje conceptual de la disciplina.

El análisis que establece las diferencias en función del sexo de cada dependencia educativa, ha puesto de manifiesto la no existencia de diferencias significativas en torno al bagaje cultural en EF entre hombres y mujeres. A pesar que son los hombres los que demuestran practicar mayormente actividad física en horario de colegio (Beltrán, Sierra, Jiménez, González-Cutre, Martínez \& Cervelló, 2017) y demuestran tener una mejor actitud positiva frente a la clase educación física (Cárcamo-Oyarzun, Wydra, Hernández-Mosqueira \& Martínez-Salazar, 2017; Fernández, González, Toja \& Carreiro, 2017). Estos resultados coinciden con las mediciones y pruebas de rendimiento deportivo, tanto internacionales como nacionales, en donde los hombres obtienen mejores resultados (Fernández, 2009), sin embargo, desde el rendimiento académico, son las mujeres las que demuestran obtener mejores resultados (López-Bonilla, López, Serra \& Ribeiro, 2015). Es por ello, Vílchez, Ruiz \& García, (2017) señalan que la actividad física debe ser intencionada en los diferentes grupos escolares, sin importar la edad, el sexo o el tipo de actividad que se quiera desarrollar.

Según los resultados obtenidos a través de la recodificación de la variable de respuestas correctas en función del percentil 75, nos permitió identificar a los estudiantes que lograban superar los 17 puntos del cuestionario. Logrando evidenciar nuevamente que los colegios particulares obtienen los mejores resultados frente al dominio disciplinar, poniendo en evidencia que estos alumnos, tienen tres veces más probabilidades de superar los 17 puntos, que los estudiantes de los colegios municipales. Estos resultados coinciden con el estudio de Bravo, Contreras \& Sanhueza (1999), a partir del SIMCE y la Prueba de Evaluación del Rendimiento Escolar (PER), donde los resultados arrojan que los establecimientos privados tienen un rendimiento más alto, y además, se distribuyen significativamente diferente a los otros tipos de dependencias, debido a un creciente aumento de la concentración de destacados alumnos académicamente en este sector.

\section{Conclusiones}

El estudio de la formación conceptual en EF, puede poner en tensión la mirada mono-focal de la disciplina. Desde esta perspectiva, es necesario generar una nueva mirada en torno al conocimiento en EF de los escolares chilenos, y así, disminuir las mediciones estandarizadas, las cuales se siguen aplicando en diferentes establecimientos educacionales.

En general, se observó un débil manejo conceptual de la disciplina en los estudiantes, poniendo de manifiesto una vez más la necesidad de buscar alternativas innovadoras para lograr satisfacer la enseñanza y aprendizaje de los con- tenidos conceptuales. En esta línea, el desafío debe situarse en torno a la búsqueda de planteamientos metodológicos que contribuyan a una mejora cuantitativa y cualitativa de la formación conceptual de los estudiantes en EF, sin que esto pueda afectar a la disminución importante del carácter práctico de las clases. Como sugerencia a esta problemática, es importante incorporar los contenidos conceptuales en las clases de una manera más lúdica y comprensiva, realizando una planificación de cada unidad didáctica para que los estudiantes trabajen, no sólo en clases, sino que también desde sus casas.

Finalmente se debe ofrecer distintas posibilidades a los estudiantes que le ayuden a conseguir un cierto nivel de conceptualización y consecución de actitudes positivas hacia la práctica sistemática de actividad física como un medio más para disfrutar de su tiempo libre.

\section{Conflicto de intereses}

Los autores no han referido ningún potencial conflicto de interés en relación con este artículo.

\section{Agradecimientos}

Este artículo se ha realizado en el contexto y gracias al proyecto de investigación Fondecyt $\mathrm{N}^{\circ} 11190537$, titulado «Evaluación del conocimiento, habilidades y actitudes de la formación pedagógica en educación física y su relación con los estilos de enseñanza y estrategias de evaluación de los académicos de la formación inicial docente», financiado por el Fondo Nacional de Investigación Científica y tecnológica de Chile.

\section{Referencias}

Agencia de Calidad de la Educación. (2013). Informe de Resultados SIMCE Educación Física $8^{\circ}$ Básico para Docentes y Directivos. Santiago de Chile. Recuperado de http:// archivos.agenciaeducacion.cl/biblioteca_digital_historica/resultados/2013/result8b_edfisica_2013.pdf

Aleixo, I., \& Mesquita, I. (2016). Impact of implementing different teaching strategies on the development of declarative knowledge of beginner artistic gymnastics athletes. Revista Brasileira de Ciências do Esporte, 38 (4); 349-357. http://dx.doi.org/10.1016/ j.rbce.2016.01.009

Almeida, F., Bracht, V. \& Vaz, A. (2012). Classificações epistemológicas na educação física: redescrições Movimento, 18(4), 241-263. https://doi.org/10.22456/19828918.27727

Ato, M. López, J. \& Benavente, A. (2013). Un sistema de clasificación de los diseños de investigación en psicología. Anales de Psicología, 29 (3), 1038-1059. http://dx.doi.org/10.6018/ analesps.29.3.178511

Beltrán, J., Sierra, A., Jiménez, A., González-Cutre, D., Martínez, C., \& Cervelló, E. (2017). Diferencias según género en el tiempo empleado por adolescentes en actividad sedentaria y actividad física en diferentes segmentos horarios del día. Retos. Nuevas Tendencias en Educación Física, Deporte y Recreación, 31. Recuperado de https://recyt.fecyt.es//index.php/retos/ article/view/36207/31342

Beltrán-Veliz, J., Aburto, B. \& Peña, S. (2018). Prácticas que obstaculizan los procesos de transposición didáctica en escuelas 
asentadas en contextos vulnerables: Desafíos para una transposición didáctica contextualizada. Revista Educación, 42 (2), 335-355. https://doi.org/10.15517/revedu.v42i2.27571

Bonilla, J., Brunner, J., Granovsky, M., Halpern, P., Iaies, G., Martínez, F. \& Tiana, A. (2003). Evaluar Las Evaluaciones: Una Mirada Política De Las Evaluaciones De La Calidad Educativa. Buenos Aires: IIPE-Unesco, 67-84.

Bracht, V. (2012). Corpo, movimento, conhecimento, educação e educação física: uma exploração filosófica. Relatório (PósDoutorado em Educação)-Programa de Pós-Graduação em Educação, Universidade Federal de Santa Catarina, Florianópolis.

Bravo, D., Contreras, D., \& Sanhueza, C. (1999). Rendimiento Educacional, Desigualdad, Y Brecha De Desempeño Privado/ Público: Chile 1982-1997. Universidad de Chile.

Cárcamo, J. (2012). El profesor de Educación Física desde la perspectiva de los escolares. Estudios pedagógicos (Valdivia), 38(1), 105-119.

Cárcamo-Oyarzun, J., Wydra, G., Hernández-Mosqueira, C., \& Martínez-Salazar, C. (2017). Actitudes hacia la educación física: Grados de importancia y conformidad según escolares de Chile y Alemania. Una mirada intercultural. Retos. Nuevas tendencias en Educación Física, Deporte y Recreación, (32), 158162.

Cardozo, J., Velasco, D., Domínguez, S., \& Reyes, J. (2019). ¿Qué se investiga en formación docente en educación física y en recreación? (What is researched in teacher training in physical education and recreation?). Retos: nuevas tendencias en educación física, deporte y recreación, (36), 3-8.

Carmona, R. (2007). ¿Cómo abordar los contenidos teóricos y actitudinales? Propuestas de acción en secundaria. Lecturas: Educación física y deportes, (104), 10. Recuperado de https:// www.efdeportes.com/efd104/contenidos-teoricossecundaria.htm

Castañer, M. \& Trigo, E. (1995). Globalidad e interdisciplina curricular en la Enseñanza Primaria: propuestas teórico-prácticas. Barcelona: Inde.

Devís, J. \& Peiró V. (1993). La actividad física y la promoción de la salud en niños/as y jóvenes. Revista de psicología del deporte, 2(2), 0071-86. Recuperado de https://www.rpd-online.com/ article/view/293/289

Díaz, J. (2012). La evaluación formativa como instrumento de aprendizaje en Educación Física. Inde.

Díaz-Barriga, F. \& Hernández, G. (2002). Estrategias docentes para un aprendizaje significativo. Una interpretación constructivista. México: Mc Graw Hill.

Díaz-Barriga, F., \& Hernández, G. (2010). Estrategias Docentes: Para un aprendizaje significativo: Una interpretación constructiva. Mc Graw Hill

Domínguez, A., Arroyo, M., Gallego, D., González, L. \& del Villar Álvarez, F. (2006). Estudio del conocimiento declarativo en función de la experiencia y de la edad en jugadores jóvenes de voleibol. Cultura, Ciencia y Deporte, 2(5), 73-80. http:// dx.doi.org/10.12800/ccd.v2i5.175

Escudero, M. (2011). Agente, subjetivación y educación corporal: reflexiones metodológicas. Revista Brasileira de Ciências do Esporte, 33(3). http://dx.doi.org/10.1590/S010132892011000300002

Fernández, M., González, M., Toja, B. \& Carreiro, F. (2017). Valoración de la escuela y la Educación Física y su relación con la práctica de actividad física de los escolares. Retos. Nuevas Tendencias en Educación Física, Deporte y Recreación, 31, 312-315. Recuperado de https://recyt.fecyt.es/index.php/retos/article/view/53508

Fernández, R. (2009). Diferencias de género en rendimiento aca- démico: efectos en la evolución a nivel escolar. (Tesis). Pontificia Universidad Católica de Chile.

Gallo, L. \& Martínez, L. (2015). Linhas pedagógicas para educação corporal. Cadernos de pesquisa, 45(157), 612-629. http:// dx.doi.org/10.1590/198053143215

Gallo, S. (2008). Deleuze \& a Educação. Belo Horizonte: Autêntica. García, L. (2016). La evaluación en la educación física escolar. Armenia: Kinesis

González, S., García, L., Pastor, J. \& Contreras, O. (2011). Conocimiento táctico y la toma de decisiones en jóvenes jugadores de fútbol (10 años). Revista de Psicología del Deporte, 20 (1), 10-32. Recuperado de https://www.rpd-online.com/article/ view/637/731

Gusthart, J. Kelly, I. \& Rink, J. (1997). The validity of the Qualitative Measures of Teaching Performance Scale as a Measure on Teacher Effectiveness. Journal of Teaching in Physical Education, 16, 196-210. https://doi.org/10.1123/jtpe.16.2.196

Hernández, J. \& López, A. (2007). Qué saben los escolares españoles. Conceptos claves en Educación Física y su evaluación. En J. L. Hernández y R. Velázquez (Coord.). La Educación Física, los estilos de vida y los adolescentes: cómo son, cómo se ven, qué saben y qué opinan, (pp.169-204). Barcelona: Graó.

Hernández, J. Velázquez, R. \& Martínez, M. (2007). Reformas Curriculares y Educación Física: los Conocimientos TeóricoConceptuales de la Población Escolar Española. Archivos Analíticos de Políticas Educativas, 15 (15), 1-31. Recuperado de https://www.redalyc.org/articulo.oa?id=275020546015

Ibañez, F. (2005). Una experiencia para el desarrollo de los contenidos conceptuales en el área de Educación Física. Lecturas: Educación Física y Deportes, 80, 35. Recuperado de https:// dialnet.unirioja.es/servlet/articulo? codigo $=1057830$

López, A. \& Moreno, J. (2002). Aprendizaje de hechos y conceptos en educación física. Una propuesta metodológica. Apunts: Educación Física y Deportes, (69), 19-26. Recuperado de https://www.revista-apunts.com/apunts/articulos//69/es/ 069_018-026ES.pdf

López, V., Pérez, D., Manrique, J. \& Monjas, R. (2016). Los retos de la Educación Física en el Siglo XXI. Retos. Nuevas Tendencias en Educación Física y Deportes, 29, 182-187.

López-Bonilla, J. M., López-Bonilla, L. M., Serra, F. \& Ribeiro, C. (2015). Relación entre actitudes hacia la actividad física y el deporte y rendimiento académico de los estudiantes universitarios españoles y portugueses. Revista iberoamericana de psicología del ejercicio y el deporte, 10(2), 275-284. Recuperado de https://www.riped-online.com/articles/relationshipbetween-attitudes-towards-physical-activity-and-sports-andacademic-performance-of-spanish-and-portuguese-c.pdf

Manzini, J. L. (2000). Declaración de Helsinki: principios éticos para la investigación médica sobre sujetos humanos. Acta bioethica, 6(2), 321-334. http://dx.doi.org/10.4067/S1726569X2000000200010

McPherson, S. (1994). The development of sport expertise: Mapping the tactical domain. Quest, 46(2), 223-240

Méndez, A. (2005). Hacia una evaluación de los aprendizajes consecuente con los modelos alternativos de iniciación deportiva. Tándem: Didáctica de la Educación Física, 17,38-58. Recuperado de https://www.researchgate.net/publication/ 39211980_Hacia_una_evaluacion_de_los_aprendizajes_c onsecuente con los modelos alternativos de iniciacion deportiva

Metzler, M. (1989). A review of research on time in sport pedagogy. Journal of Teaching in Physical Education, 8, 87-103. https:// doi.org/10.1123/jtpe.8.2.87

Mizala, A., \& Romaguera, P. (1998). ¿Cómo se comparan los resultados de la prueba SIMCE entre colegios privados y públicos? Perspectivas en Política, Economía y Gestión, 2(1), 107-130. 
Moreno, A. \& Medina, J. M. (2012). Número Especial: Escuela, educación física y transformación social. Estudios pedagógicos (Valdivia), 38 (ESPECIAL), 7-11.

Moreno, A., Campos, M. \& Almonacid, A. (2012). Las funciones de la educación física escolar: una mirada centrada en la justicia social y la reconstrucción del conocimiento. Estudios pedagógicos, 13-26. http://dx.doi.org/10.4067/S071807052012000400002

Moreno, A., Toro-Arévalo, S. \& Gómez, F. (2020). Formación inicial de maestros de educación física: conectando un quehacer pedagógico decolonial con la intervención social, política e insurgente del espacio público. Retos Nuevas tendencias en Educación Física, Deportes y recreación. Nº37, 1988-2041. Recuperado de https://recyt.fecyt.es/index.php/retos/article/view/ $74183 / 0$

Otero, F. González, J. Calvo, A. \& Molina, E. (2012). Contenidos conceptuales en Educación Física: efecto de un programa de intervención en el primer ciclo de primaria. Revista Digital de Educación Física, (16), 55-68. Recuperado de https:// dialnet.unirioja.es/servlet/articulo? codigo $=3929976$

Páez, J. \& Hurtado, J. (2019). Formación inicial docente en profesores de educación física. Levantamiento de competencias específicas a partir de las necesidades del medio educativo. Retos. Nuevas Tendencias en Educación Física y Deportes, 35, 61-66

Peña, S. \& Hernández, J. (2014). Evaluación del conocimiento teórico-conceptual en Educación Física en escolares Chilenos de enseñanza media de la ciudad de Puerto Montt. Revista horizonte ciencias actividad física, 5(1), 65-72. Recuperado de http://www.revistahorizonte.ulagos.cl/index.php/horizonte/ article/view/19

Peña, S., Osses, S., Navarro, B. \& Beltrán, J. (2017). Hacia la disminución de los test físicos como indicadores de salud en el contexto educativo. Salud Pública de México, 59(5), 506-507. https://doi.org/10.21149/8054

Peña, S., Toro, S., Osses, S. \& Hernández-Mosqueira, C. (2018) Diseño y validación de un cuestionario para evaluar la dimensión conceptual en educación física. Sport TK: revista euroamericana de ciencias del deporte, 7(2), 91-100. https:// doi.org/10.6018/sportk.342991

Peña, S., Toro, S., Osses, S., Moreno, J. \& Hernández-Mosqueira, C. (2019). La Dimensión Conceptual del Conocimiento en Educación Física: Un estudio Binacional. Retos: nuevas tendencias en educación física, deporte y recreación, (35), 170175. Recuperado de https://recyt.fecyt.es/index.php/retos/ article/view/63164

Poblete, F., Linzmayer, L., Matus, C., Garrido, A. \& Flores, C. (2018). Percepción de estudiantes de Pedagogía en Educación Física hacia sus profesores. Retos. Nuevas Tendencias en Educación Física y Deportes, 33, 143-147.

Rodríguez, F., Coz, D., Durán, T., Guajardo, A., Alvarado, C. \& Doña A. (2015). Sistema de medición de la calidad de la educación física en Chile y su influencia en la realidad escolar. Movimento, 21(2): 435-448. Recuperado de https://seer.ufrgs.br/ Movimento/article/viewFile/49693/34223

Rodríguez, P., \& Hernández, F., (2018). Didáctica de la Motricidad en la formación de profesores de educación infantil. Retos: nuevas tendencias en educación física, deporte y recreación, (34), 25-32.

Rodríguez-Rodríguez, F., Curilem, C., Escobar, D. \& Valenzuela, L. (2016). Propuesta de evaluación de la educación física escolar en Chile. Educación Física y Ciencia, 18(1), 00-00. Recuperado de https://www.efyc.fahce.unlp.edu.ar/article/view/ EFyCv18n01a03/7406

Romero-Martín, R., Castejón-Oliva, F. \& López-Pastor, V. (2015). Divergencias del alumnado y del profesorado universitario so- bre las dificultades para aplicar la evaluación formativa. RELIEVE. Revista Electrónica de Investigación y Evaluación Educativa, 21(1). https://doi.org/10.7203/relieve.21.1.5169

Ruiz, L., Sánchez, M., Durán, J., \& Jiménez, C. (2006). Los expertos en el deporte: Su estudio y análisis desde una perspectiva psicológica. Anales de psicología, 22(1). Recuperado de https:/ /revistas.um.es/analesps/article/view/26671

Sánchez, G. \& López, M. (2019). Análisis de los contenidos de expresión corporal impartidos en la formación inicial de los docentes de primaria. Educación XX1, 22(1), 425-447. https:/ /doi.org/10.5944/educXX1.20058

Scheaffer, R., Mendenhall, W. \& Ott, L. (1987). Elementos de muestreo (México D.F., México: Grupo Editorial Iberoamérica.

Silverman, S., Devillier, R. \& Ramírez, T. (1991). The validity of academic learning time-physical education (ALT-PE) as a process measure of achievement. Research Quarterly for Exercise and Sport, 62(3), 319- 325. doi: https://doi.org/ 10.1080/02701367.1991.10608729

Thomas, K., \& Thomas, J. (1994). Developing expertise in sport: The relation of knowledge and performance. International Journal of Sport Psychology, 25, 295-295.

Tobar, B., Gaete, M., Lara, M., Pérez, A., \& Freundt, A. (2019). Teorías implícitas y modelos de formación subyacentes a la percepción de rol del profesor de educación física (Implicit theories and models of formation underlying the perception of the role of the teacher of physical education). Retos, 36(36), 159-166.

Toro, S., Pazos, J., Vargas, E. \& Vega, J. (2016). De la educación global a la biología del conocer: Comprender el sentido de la educación. Estudios pedagógicos (Valdivia), 42 (ESPECIAL), 57-73. http://dx.doi.org/10.4067/S0718-07052016000300006

Trigueros, C., Rivera, E. \& Moreno, A. (2020). The Big Bang Theory o las reflexiones finales que inician el cambio. Revisando las creencias de los docentes para construir una didáctica para la Educación Física Escolar. Retos: Nuevas Tendencias en Educación Física, Deportes y Recreación. 37(37). Recuperado de https://recyt.fecyt.es/index.php/retos/article/view/74175

Velázquez, R., Hernández, J., Martínez, M. \& Martínez de Haro, V. (2009). Educación Física y conocimiento teórico-conceptual: estudio trans-cultural en Argentina, Brasil y España. Revista de Educación, 356, 653-675. https://doi.org/10.15366/ riee2019.12.1.012

Vera, J. A. \& Moreno, J. A. (2016). Razones intrínsecas para la disciplina en estudiantes adolescentes de Educación Física. Educación XX1, 19(2) http://dx.doi.org/317-335. 10.5944/ educXX1.16469

Vílchez, M., Ruiz-Juan, F. \& García, M. (2017). Estudio transcultural de la percepción de competencia escolar y tiempo de ocio. Revista Internacional de Medicina y Ciencias de la Actividad Física y el Deporte, vol. 17 (67) pp. 573-587. https:/ /doi.org/10.15366/rimcafd2017.67.012

Whitehead, M. (2010). Physical literacy: Throughout the lifecourse. Routledge.

Zabala, M., Viciana, J. \& Lozano, L. (2002). La planificación de los deportes en la educación física de ESO Lecturas: Educación Física y deportes. Revista Digital, 8, 48. Recuperado de https:/ /www.efdeportes.com/efd48/eso.htm

Zabala, V. (2000). «El aprendizaje de los contenidos según su tipología» en La práctica educativa. Cómo enseñar. 7a ed. España: Graó.

Zaragozá, J. \& Martínez, A. (2018). Conectando teoría y práctica profesional en el Grado en Ciencias de la Actividad Física y el Deporte. Revista Española de Educación Física y Deportes, 420,39-57. 